\title{
Rursus
}

Russus Poiétique, réception et réécriture des textes antiques

$1 \mid 2006$

Le modèle animal (I)

\section{Enquête sur les doctorats et les HDR}

\section{Evrard Delbey et Robert Alessi}

\section{OpenEdition}

Journals

Édition électronique

URL : http://journals.openedition.org/rursus/55

DOI : 10.4000/rursus.55

ISSN : 1951-669X

\section{Éditeur}

Université Nice-Sophia Antipolis

\section{Référence électronique}

Evrard Delbey et Robert Alessi, «Enquête sur les doctorats et les HDR », Rursus [En ligne], 1 | 2006, mis en ligne le 09 juillet 2007, consulté le 19 avril 2019. URL : http://journals.openedition.org/ rursus/55; DOl : 10.4000/rursus.55

Ce document a été généré automatiquement le 19 avril 2019

Rursus 


\title{
Enquête sur les doctorats et les HDR
}

\author{
Evrard Delbey et Robert Alessi
}

1 Nous remercions chaleureusement les collègues qui nous ont adressé leurs réponses. Nous commencerons par citer les réponses... sans réponses en ne voulant pas toutefois entamer un quelconque chant élégiaque sur des études classiques inexorablement tombées au champ d'honneur. Ainsi, Angers sans doctorants «Lettres classiques », Le Mans privé de cursus "Lettres classiques » et Orléans, Valenciennes existent tout de même encore ; Pau est parvenu à mettre en place depuis deux ans un cursus «Lettres classiques » jusqu'aux concours de l'enseignement. A signaler la situation de l'E.N.S. Lyon où l'année universitaire 2004-2005 est la première durant laquelle l'Ecole est susceptible de délivrer des Doctorats en cohabilitation avec Lyon 2 et Lyon 3.

\section{Questions sur les Doctorats}

2 - Nous renvoyons au Tableau ci-joint pour les trois premières questions à propos du nombre d'inscrits en Latin, en Grec et en Linguistique ancienne, pour l'évaluation de la durée moyenne des Doctorats et pour le nombre de soutenances. Nous avons préféré ne pas fixer les années universitaires de référence, sachant que le dernier quadriennal est un repère commode et surtout que nos informations dépendent le plus souvent d'enquêtes internes à chaque Université qui ne sont pas toujours très récentes ou disponibles immédiatement parce qu'en cours de réalisation.

3 - La quatrième question portait sur le statut des Doctorants. Les réponses reçues font surtout état des Allocataires Moniteurs Normaliens, des Allocataires Moniteurs, des ATER et des enseignants du second degré notamment présents à Aix, Bordeaux 3, Brest, Clermont-Ferrand, Grenoble, Limoges, Lyon 2, Paris12, Strasbourg 2 et Tours. Il nous semble que reste ouverte la question du nombre de Doctorants du second degré soutenant leur thèse; par exemple, les trois Doctorants inscrits à Poitiers en 2003, et ayant abandonné, étaient du Secondaire. Cet exemple prend-il valeur à l'échelle nationale?

4 - La cinquième question portait sur une éventuelle préférence pour l'inscription en Doctorat sans succès préalable à un concours de l'enseignement. Apparemment, le 
problème ne se pose pas encore; les cas d'étudiants de master ou désireux de devenir Allocataires Moniteurs et commençant par la préparation d'un Doctorat sont isolés; lorsque l'Allocataire Moniteur est d'origine étrangère, il n'a pas besoin d'un concours français pour obtenir un poste dans son pays.

\section{Enquête sur les Doctorats}

\section{Tableau des résultats}

\begin{tabular}{|c|c|c|c|c|c|}
\hline Université & $\begin{array}{l}\text { Doctorats } \\
\text { inscrits en } \\
\text { Latin }\end{array}$ & $\begin{array}{l}\text { Doctorats } \\
\text { inscrits en } \\
\text { Grec }\end{array}$ & $\begin{array}{l}\text { Doctorats inscrits } \\
\text { en Linguistique }\end{array}$ & $\begin{array}{l}\text { Durée } \\
\text { moyenne }\end{array}$ & $\begin{array}{l}\text { Nombre de } \\
\text { soutenances }\end{array}$ \\
\hline Aix & 13 & 13 & 0 & 6 ans & $?$ \\
\hline Bordeaux 3 & 6 & 5 & 0 & $4 / 5$ ans & 1 ou 2 an(s) \\
\hline Brest & 0 & 4 & 0 & \pm 4 ans & $1(2002)$ \\
\hline $\begin{array}{l}\text { Clermont- } \\
\text { Fd }\end{array}$ & 0 & 4 & 1 (latine) & $5 / 6$ ans & $3(2002-2004)$ \\
\hline Grenoble & 3 & 1 & 0 & \pm 6 ans & $2(2000-2004)$ \\
\hline Limoges & 2 & 0 & 0 & $?$ & 0 \\
\hline Lyon 2 & 3 & 7 & 7 & 5 ans & 7 (2002-2005) \\
\hline Lyon 3 & 2 & 1 & 0 & $4 / 5$ ans & $2(2004)$ \\
\hline Nice & 0 & 0 & 1 & $4 / 5$ ans & $2(2003-2005)$ \\
\hline Paris 4 & 49 & 73 & 53 & $4 / 5$ ans & $1(2003-2005)$ \\
\hline Paris 12 & 5 & 2 & 0 & $?$ & $4(2003-2005)$ \\
\hline Poitiers & 0 & 0 & 0 & $?$ & 0 \\
\hline $\begin{array}{l}\text { Strasbourg } \\
2\end{array}$ & 9 & 11 & 0 & $4 / 5$ ans & 6 (2003-2005) \\
\hline Toulouse & 2 & 4 & 0 & 3 ans & $5(2001-2004)$ \\
\hline Tours & 4 & 2 & 0 & 4 ans & $2(2004)$ \\
\hline
\end{tabular}

\section{Questions sur les H.D.R.}

5 - La première question avait pour sujet le moment du choix d'un directeur ou tuteur. « Le plus tôt possible ", autrement dit dès le début du travail devant aboutir à l'Habilitation, et 
« au dernier moment », autrement dit lorsque le passage de l'H.D.R. est vraiment décidé, sont les deux extrêmes des réponses collectées; dans la zone intermédiaire, trois à deux ans avant la soutenance est vu comme un juste milieu. A vrai dire, la réponse strictement administrative fixe le choix un an avant la soutenance, mais ce qui importe est la réponse pédagogique et scientifique.

6 - La seconde question avait pour objet la durée moyenne de l'H.D.R. Entre cinq et dix ans après l'obtention du Doctorat (Aix et Lyon 2), sept à huit ans (Clermont-Ferrand), deux à cinq ans (Grenoble), huit à dix ans (Strasbourg 2), trois à dix ans (Toulouse). L'obligation d'inscription ne valant que l'année de soutenance, les durées estimées sont très variables.

7 - La troisième question portait sur le nombre de soutenances. Les chiffres que nous avons relevés sont les suivants :

\begin{tabular}{|l|l|}
\hline Bordeaux 3: & 0 \\
\hline Clermont-Ferrand : & 0 \\
\hline Grenoble : & $1(2005)$ \\
\hline Lyon 2: & $2(2003)+1(2004)$ \\
\hline Lyon 3: & 0 \\
\hline Nice : & 0 \\
\hline Paris 4: & $46(2003-2004)$ \\
\hline Paris 12: & 3 (dernier quadriennal) \\
\hline Strasbourg 2: & 1 \\
\hline Toulouse : & 1 (2001) \\
\hline Tours : & 2 (2004). \\
\hline
\end{tabular}

8 - La quatrième question avait trait au règlement de chaque Université pour la composition du dossier d'Habilitation. Les situations sont variées pour ne pas dire disparates...

9 Aix fixe cinq éléments : 1) Présentation synthétique des travaux, de la méthode de recherche, des activités d'enseignement et des coopérations de recherches, des projets 2) Ouvrages publiés 3) Recueil des articles publiés 4) Mémoire original -étude, édition, traduction, commentaire 5) Recueil d'inédits éventuellement ou publications annexes diffusion du savoir ;

Bordeaux 3 indique: un CV + travaux, articles, ouvrages, thèse avec le rapport de soutenance + synthèse des travaux ou plan détaillé permettant de faire apparaître l'expérience du candidat dans « l'animation d'une recherche » (au moins quarante pages) + une lettre de recommandation d'un Professeur de Bordeaux 3 ;

11 Brest fait notamment état de : vingt-cinq pages à remettre au Conseil Scientifique, avec $\mathrm{CV}$, liste des publications, présentation des travaux et des projets à quatre ans 
(transmission sous forme d'un fichier électronique et de trois exemplaires papier), une liste de cinq spécialistes francophones de notoriété nationale et internationale susceptibles de juger le travail; le dossier complet pour la soutenance comprend les publications du candidat, les travaux non publiés encore et une synthèse ;

Clermont-Ferrand mentionne : l'ensemble des travaux dont, si possible, la thèse publiée, un document de synthèse des travaux de cinquante pages + une monographie originale ;

13 Grenoble insiste sur le débat qui doit d'abord avoir lieu entre le "directeur » et le "candidat" à propos de la composition du dossier scientifique, sur le mémoire de synthèse de soixante-dix pages au moins et sur «les traditions propres à notre discipline »; insistance également sur la bonne mise en évidence de ce qui est nouveau par rapport à la thèse, sur les améliorations apportées à celle-ci pour sa publication, sur la mention des recensions dont la thèse publiée a fait l'objet, des comptes rendus publiés par le candidat lui-même, sur les publications à caractère pédagogique, un chercheur étant aussi un enseignant qui doit avoir le souci de s'adresser à un large public ;

Lyon 2 mentionne : un volume de soixante-dix pages minimum de synthèse + les travaux dont un inédit ;

Lyon 3 mentionne : un CV détaillé (cursus universitaire, activités professionnelles dont activités d'enseignement et de recherche, encadrement de mémoires...), la liste des publications, interventions dans des colloques, contrats de recherche du candidat depuis la soutenance du Doctorat, un recueil d'ouvrages, d'articles, un rapport synthétisant les recherches entreprises par le candidat depuis son Doctorat, enfin la copie du rapport de soutenance de thèse ;

16 Nice s'en tient à un $\mathrm{CV}$ avec une synthèse de l'activité scientifique permettant de faire apparaitre l'expérience du candidat dans « l'animation d'une recherche » et à la demande de l'ensemble des travaux et publications postérieurs à ceux du Doctorat

17 Paris 4 fait figurer notamment l'avis du Directeur de Recherche, un CV, un résumé sur le thème de la recherche, le rapport de soutenance de la thèse, la liste des publications scientifiques et, dans le dossier strictement scientifique, un ouvrage original dactylographié, l'ensemble des travaux scientifiques, le mémoire de synthèse de l'activité scientifique du candidat;

18 Paris 12 constate qu'il n'y a pas de définition interne stricte pour la composition du dossier, si ce n'est pour la présence du document de synthèse ;

19 Strasbourg 2 énumère les travaux + le document de synthèse ; trois rapports scientifiques + avis du directeur d'H.D.R. ;

20 Toulouse demande le CV + la liste des travaux (articles et ouvrages), les travaux euxmêmes et la synthèse scientifique ;

21 Tours enfin insiste sur le mémoire de synthèse, le dossier des travaux réalisés depuis la thèse et sur la publication de la thèse de Doctorat.

22 - La cinquième et dernière question se rapportait aux éléments du dossier pour l'H.D.R. faisant problème. Nous avons relevé le volume de synthèse à la définition jugée imprécise (il n'est pas toujours lu par les membres du jury), les publications dont le nombre n'est pas non plus précisé, la thèse de Doctorat elle-même dont la publication n'est pas exigée explicitement. Quant à la rédaction d'un ouvrage original, elle peut s'avérer difficile. 


\section{Questions sur les Doctorats et les H.D.R.}

23 - La première question portait à la fois sur la durée des Doctorats et des H.D.R. dans les disciplines autres que les Lettres classiques et sur le nombre des soutenances dans ces mêmes disciplines, lorsque l'Ecole Doctorale est ouverte à plusieurs disciplines. Durées très comparables (Clermont-Ferrand, Lyon 2, Lyon 3 précise: cinq ans environ, Tours), alors que Poitiers note que la durée de préparation est assez brève, trois ans, pour les Doctorats de Géographie, de Psychologie cognitive, de Sciences économiques... et que Strasbourg indique une durée plus longue en Lettres, Histoire, Philosophie (cinq à six ans), moins longue en Sciences sociales et Linguistique (trois ans) et intermédiaire pour les Langues. Toulouse insiste sur le fait que dans les disciplines autres que les Lettres classiques, mise à part l'Histoire, la durée des Doctorats est actuellement de trois ans le plus souvent. Brest note que lorsque les Doctorants sont salariés, la durée peut aller jusqu'à sept ou huit ans. Le nombre d'inscriptions est plus élevé dans la plupart des autres disciplines (Brest, Clermont-Ferrand, Nice) ainsi que le nombre de soutenances (Bordeaux 3, Brest, Grenoble, Lyon 3, Nice, Paris 12, Poitiers, Strasbourg 2) alors qu'ils sont sensiblement identiques à Lyon 2 et que le nombre de soutenances est tout à fait comparable à Clermont-Ferrand.

24 - La seconde question visait la nature des Ecoles Doctorales. Pluridisciplinaires et locales majoritairement (Aix -«Espaces, cultures et sociétés »-, Bordeaux 3 -«Histoire/ Géographie »-, Brest -« Lettres, Langues, Sociétés, Gestion »- Clermont-Ferrand -« Lettres et Sciences Humaines et Sociales »-, Grenoble sans intitulé défini, Limoges -« Sciences de l'Homme et de la Société »-, Lyon 2 -«Humanités et Sciences Humaines »-, Lyon 3 -«Systèmes, Images, Langages »-, Nice -«Lettres, Sciences Humaines et Sociales», Poitiers - « Sciences Humaines, Economiques et Sociales »-, Paris 4 -« Mondes anciens et médiévaux »-, Paris 12 «Philosophie et littérature. Histoire, doctrines, expressions »-, Strasbourg 2 -«Ecole Doctorale des Humanités »-Toulouse -«Temps. Espace. Sociétés. Cultures», Tours - "Histoire des représentations»), les Ecoles Doctorales auxquelles appartiennent les collègues qui nous ont répondu les satisfont globalement même si la défense de nos disciplines n'est pas aisée dans ces vastes ensembles où la répartition d'allocations de recherche allouées par le Ministère ne nous est pas favorable. L'argument principal en leur faveur est qu'il faut éviter le clivage entre " petites universités ", privées d'Ecole Doctorale, et "grandes universités » devenues pôles d'excellence et attirant à elles un plus grand nombre d'étudiants préférant une préparation plus spécialisée au sein d'une monodisciplinarité interuniversitaire. Les partenaires privilégiés sont les historiens et les archéologues -sciences de l'Antiquité- ainsi que les collègues de Lettres modernes et de Littérature comparée.

25 - La troisième question s'intéressait au calendrier des soutenances. Le choix de la date de soutenance est libre. Cependant le début des soutenances en septembre apparaît souhaitable ; il s'agit pour les candidats d'être prêts...

26 - La dernière question avait rapport à la possibilité de publier « en ligne »; les avis sont partagés : reconnaissant la rapidité et la facilité d'accès aux publications électroniques, nos collègues souvent restent attachés aux livres d'un maniement plus confortable et moins transitoire. La question de la protection intellectuelle est posée pour une pratique qui nécessite un comité éditorial sur site reconnu. 


\section{AUTEURS}

EVRARD DELBEY

Université de Nice

ROBERT ALESSI

Université de Poitiers 